\title{
Association of Metabolic Syndrome with Vitiligo- A Case Control Study
}

\author{
P. K. Sinha ${ }^{1}$, Prashant Nigam² J. P. Swain ${ }^{3}$
}

${ }^{1}$ Assistant Professor, Department of DVL, GMC, Ambikapur, Chhattisgarh, India. ${ }^{2}$ Associate Professor, Department of Biochemistry, CIMS, Bilaspur, Chhattisgarh, India. ${ }^{3}$ Professor, Department of DVL, CIMS, Bilaspur, Chhattisgarh, India.

\section{ABSTRACT}

\section{BACKGROUND}

Hypertension, abdominal obesity, dyslipidaemia, glucose intolerance are collectively termed as metabolic syndrome which leads to further health problems such as inflammatory skin diseases. Many recent studies suggest that its association with skin disorders was due to oxidative stress. Vitiligo is one of the common skin inflammatory diseases. In the present study, we wanted to evaluate the association of metabolic syndrome with Vitiligo.

\section{METHODS}

In the present study, we selected 75 subjects attending skin OPD with age matched 75 controls and assessed the waist circumference, blood pressure, serum triglyceride level, cholesterol and high-density cholesterol along with glucose at Chhattisgarh Institute of Medical Sciences, Bilaspur. A detailed history including age, gender, diabetes mellitus, hypertension, smoking and onset of vitiligo was taken. General physical examination including recording of weight, height, body mass index (BMI), blood pressure and waist circumference was done. Fasting venous blood sample was taken and vitiligo disease activity (VIDA) score was obtained. The MetS criteria were defined by National Cholesterol Education Program Adult Treatment Panel III 2005 (ATP III) guidelines.

\section{RESULTS}

We found significant differences in Serum HDL $(\mathrm{p}<0.001)$, and Triglyceride levels ( $\mathrm{p}<0.001$ ) only. In our study, there was no significant difference in waist circumference, plasma glucose and blood pressure between cases and controls.

\section{CONCLUSIONS}

We observed interrelation between Vitiligo and development of metabolic syndrome. The metabolic syndrome is somehow related with Vitiligo. Literature in this regard is less available, further study is required to establish the relation between Vitiligo and Metabolic Syndrome.

\section{KEY WORDS}

Metabolic Syndrome, Vitiligo, Hyperlipidaemia
Corresponding Author:

Dr. Prashant Nigam,

Associate Professor,

Department of Biochemistry,

CIMS, Bilaspur, Chhattisgarh, India.

E-mail: nigam.prashant866@gmail.com

DOI: 10.14260/jemds/2019/604

Financial or Other Competing Interests: None.

How to Cite This Article:

Sinha PK, Nigam P, Swain JP. Association of metabolic syndrome with vitiligo- a case control study. J. Evolution Med. Dent. Sci. 2019;8(36):2783-2786,

10.14260/jemds/2019/604

Submission 09-07-2019,

Peer Review 24-08-2019,

Acceptance 31-08-2019,

Published 09-09-2019.

\section{(i) $(5$}

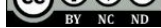




\section{BACKGROUND}

Metabolic syndrome includes hypertension, dyslipidaemia, abdominal obesity and glucose intolerance. ${ }^{1}$ This syndrome is often associated with other systemic disorders. Recently many studies suggested its association with skin disorders with oxidative stress. ${ }^{2}$ Vitiligo in one of the progressive depigmenting skin disorder believed to be due to genetic, immunological, cytotoxic and inflammatory factors about 02 $\%$ of the world population is affected by vitiligo. In Vitiligo the patches formed are with sharp margins may also involve mouth and nose. Pathology of Vitiligo is unknown and believed to be due to genetic susceptibility. It is classified into two classes segmental and non-segmental. There is a probable symmetry is always present in the location of patches in non-segmental type of Vitiligo. This class includes generalized, Universal, Focal, Acrofacial and Mucosal Vitiligo. Later one is static and mostly affected the area of skin attached with dorsal root of spinal cord unilaterally. Approximately $20 \%-25 \%$ of world population are having increased risk to develop cardiovascular disorders and stroke associated with metabolic syndrome. Metabolic syndrome depends on obesity, daily routine, aging and genetic factors. The criteria for the diagnosis of MetS depends on waist circumference, Blood Pressure, Hyperglycaemia and Hyperlipidaemia along with low HDL. (Table 1) Metabolic syndrome depends on various factors can be classified as underlying factors, major factors and emerging risk factors. Abdominal obesity, less physical activity, high fat containing diet are the underlying factors whereas use of tobacco as smoking, high blood pressure, increased LDL and cholesterol and low HDL levels are the major risk factors for the MetS. Insulin resistance and glucose intolerance are the emerging risk factors. It is documented in literature that oxidative stress is one of the main reasons for the pathogenesis of Metabolic Syndrome, possible related with Vitiligo. ${ }^{3}$ The lipid disturbances in Vitiligo due to disturbed metabolic processes in the adipose tissue and oxidative stress causes Metabolic syndrome. Insulin activity is adversely affected by increased expression of IL-6, IL-1 or TNF-a which further expressed as insulin resistance, endothelial dysfunction and finally development of Metabolic Syndrome. Reactive Oxygen Species are naturally produced during oxidative metabolism of cell and affects the physiological processes of cell like including inflammation. 4,5 Reactive oxygen species includes free radicals e.g. superoxide $\left(\mathrm{O}_{2} \bullet-\right)$ non radical e.g. $\mathrm{H}_{2} \mathrm{O}_{2}$ etc. Antioxidant enzymes like superoxide dismutase scavenge the $\mathrm{O}_{2} \bullet$ - into $\mathrm{H}_{2} \mathrm{O}_{2}$. Superoxide ion causes lipid peroxidation and formation of malonaldehyde. ROS are normally produced in body during various physiological processes and wellregulated by antioxidant system which includes enzymes, flavonoids, Vit C, VitE Glutathione etc. ${ }^{6}$ Imbalance in the above system known as oxidative stress. Significant correlation between cell physiology, ROS production and Metabolism is well documented. Recently a hypothesis is proposed regarding anti-inflammatory effects of melanocytes and scavenging of reactive oxygen species. ${ }^{7}$ Melanocytes thus reduced the reactive oxygen species and may delay the initiation of inflammation conditions ultimately metabolic syndrome. The number of melanocytes is decreased in Vitiligo patients and it has been suggested that with less number of melanocytes the probability of Metabolic syndrome is increased. ${ }^{8}$ The relation between Vitiligo and metabolic syndrome is not well documented in literature. This study was done to establish relation between Vitiligo and Metabolic Syndrome.

\section{METHODS}

This case-control study was conducted on Vitiligo patients attending the OPD of the Dermatology, Venereology and Leprosy Department of Chhattisgarh Institute of Medical Sciences, Bilaspur. A total of 150 individuals were included 75 Vitiligo patients and 75 individuals as controls. The sample size was taken based on the convenience of the study. The control group included healthy, age-matched and sexmatched volunteers who had no dermatological or systemic diseases. Pregnant and lactating women, patients less than 18 years were excluded. A detailed history including age, gender, diabetes mellitus, hypertension, smoking, onset of vitiligo were taken. General physical examination including recording of weight, height, body mass index (BMI), blood pressure and waist circumference was done. Fasting venous blood sample was taken and vitiligo disease activity (VIDA) score was obtained. The MetS criteria were defined by National Cholesterol Education Program Adult Treatment Panel III 2005 (ATP III) guidelines. ${ }^{9}$ Got approval from IEC and consent was taken from all patients.

\section{Statistical Analysis}

Data were analysed by counting mean and standard deviation and compared with each other by considering $p$ Value $<0.001$ significant.

\section{RESULTS}

\begin{tabular}{|c|c|}
\hline Diagnostic Criteria (Any 3 Below) & Value \\
\hline \multirow{2}{*}{ Obesity } & Waist circumference \\
\cline { 2 - 2 } & $\mathrm{BMI} \geq 30 \mathrm{~kg} / \mathrm{m}^{2}$ \\
\cline { 2 - 2 } & $\mathrm{Ethnic} / \mathrm{Race}$ \\
\hline Triglycerides & $>150 \mathrm{mg} / \mathrm{dL}$ \\
\hline \multirow{2}{*}{ HDL cholesterol } & Men $<40 \mathrm{mg} / \mathrm{dL}$ \\
\hline Blood pressure & $130 / 85 \mathrm{~mm} \mathrm{Hg}(\mathrm{Systolic} /$ Diastolic) \\
\hline fasting glucose & $>100 \mathrm{mg} / \mathrm{dL}$ \\
\hline Table 1. Criteria for Metabolic Syndrome \\
\hline
\end{tabular}

\begin{tabular}{|c|c|c|c|c|}
\hline $\begin{array}{c}\text { Sl. } \\
\text { No. }\end{array}$ & Parameters & $\begin{array}{c}\text { Control } \\
(\text { Mean } \pm \text { SD) }\end{array}$ & $\begin{array}{c}\text { Cases } \\
(\text { Mean } \pm \text { SD) }\end{array}$ & $\begin{array}{c}\text { p Value } \\
(\text { Mean } \pm \text { SD) }\end{array}$ \\
\hline 1. & Fasting Blood Sugar & $98 \pm 19.67$ & $101.56 \pm 14.3$ & 0.087 \\
\hline 2. & Serum Cholesterol & $190.64 \pm 24.4$ & $198.23 \pm 28.1$ & 0.095 \\
\hline 3. & Serum Triacylglycerol & $142.59 \pm 29.3$ & $184.21 \pm 59.4$ & $<0.001$ \\
\hline 4. & Low Density Lipoprotein & $89.65 \pm 12.1$ & $123 \pm 19.32$ & $<0.001$ \\
\hline 5. & High Density Lipoprotein & $46.89 \pm 17.2$ & $39.45 \pm 12.6$ & $<0.001$ \\
\hline 6. & Waist Circumference & $84.45 \pm 09.5$ & $81.35 \pm 10.2$ & 0.043 \\
\hline 7. & Systolic Blood Pressure & $116 \pm 13.4$ & $118.4 \pm 11.5$ & 0.56 \\
\hline 8. & Diastolic Blood Pressure & $78.4 \pm 5.8$ & $79.4 \pm 7.1$ & 0.064 \\
\hline \multicolumn{5}{|c|}{ Table 2. Values Observed in Cases and Controls } \\
\hline
\end{tabular}

In our study total 75 vitiligo patients were assessed with 50 age matched control subjects. Fasting Glucose, Cholesterol Triacylglycerol, LDL, HDL, Waist Circumference, Systolic and diastolic blood pressure were measured. There was no significant difference was observed in Fasting Glucose level in cases $(101.56 \pm 14.3)$ when compared to control. Waist 
Circumference $(81.35 \pm 10.2)$ was also with no any significant difference when compared to control. Value of Serum cholesterol $(198.23 \pm 28.1)$ was also without any agreement with level of Cholesterol of normal subject. In Vitiligo patients the level of serum Triacylglycerol was found higher $(184.21 \pm 59.4)$ and shows highly significant when compared with control. The levels of HDL $(39.45 \pm 12.6)$ in Vitiligo patients were highly significant when compared with control. LDL $(123 \pm 19.32)$ were presented highly significant when compared with control. There was also no significant difference was observed in blood pressure systolic and diastolic both.

\section{DISCUSSION}

Vitiligo is relatively common disorder in spite of races affected all. Gujarat and Maharashtra are highly reported state in India. Few dermatological outpatient records show, the incidence of Vitiligo to be $0.25 \%-25 \%$ in India. The mean age of onset of vitiligo is 20 years for male and 25 years for female. The etiopathogenesis of Vitiligo is complex, multifactorial and largely unknown ${ }^{10}$. Melanocytes contains enzymatic and non-enzymatic antioxidants including Superoxide dismutase, Glutathione Peroxidase, Catalase, Ascorbic acid etc. Antioxidants of melanocytes maintain optimal redox balance and prevent oxidative damage. ${ }^{11}$ In vitiligo patient death of melanocytes is believed to be due to oxidative stress. ${ }^{12}, 13$ Mitochondria is the main site of the production of Reactive Oxygen Species as a end product of normal aerobic metabolism. Apoptosis, inflammation and differentiation are also regulated by ROS. Excess generation of ROS leads to DNA injury which may cause to cell death and other pathology. The intrinsic antioxidant defence system protects cell from these injuries. Enzymes as SOD, Catalase and Glutathione peroxidase are the principal elements of the defence system. Production of Superoxide ion and hydrogen peroxide is due to synthesis of melanin causes oxidative stress to melanocytes. Activity of enzyme tyrosinase on tyrosine produces dopaquinone which reacts with thiols or amino acids and finally generates superoxide ions. Quinones forms from redox cycling of indoles also generate reactive oxygen species. In Vitiligo patients, melanocytes cannot properly cope with oxidative stress.

Melanocytes also destructed by very high level of $\mathrm{H}_{2} \mathrm{O}_{2}$ along with reduced activity of catalase in the epidermis of Vitiligo patients. ${ }^{14}$ Its also oxidizing other enzymes like glutathione peroxidase and reductase. Metabolic syndrome is a condition in which mainly insulin resistance is developed and ultimately leads to cardiovascular complications. The factors precipitated metabolic syndromes are smoking, increasing age, Obesity, Low Socioeconomic status, physical unactivity etc. Insulin hormone produced by beta cell of pancreas regulates the glucose uptake, amino acid uptake, protein synthesis, proteolysis, lipolysis, and endogenous glucose production. Obese persons are more prone to develop MetS. Visceral fat as well as subcutaneous fat both are associated with development of MetS. Increased blood pressure also has significant role in MetS. Hypertension is often related with obesity. Insulin resistance is one of the main causes. MetS patients often present with High Cholesterol, LDL and low HDL levels. Impaired fasting glucose in MetS is also associated with insulin resistance. Impaired insulin signalling starts deposition of fat with rich cholesterol core. Dyslipidaemia manifested as high level of free fatty acid, Triglyceride, LDL in Mets. These consequences are probably due to insulin resistance. Inadequate esterification results in less fatty acid trapping and ultimately reduced retention in adipose tissues increases free fatty acids in circulation. This increased free fatty acid reached peripheral to hepatic tissues. Which with insulin resistant state stimulate liver triglyceride synthesis. This may be one of the causes for increased TG level in Mets. Hypertriglyceridemia causing abnormality in LDL. It is observed that small dense LDL in only seen when triglyceride level exceeded $1.5 \mathrm{mmol} / \mathrm{l}$. When Insulin does not exert its effect due to less sensitivity of the cell causes more release of Insulin to achieve glucose balance and ultimately develop the pathological condition known as Type 2 Diabetes mellitus. Insulin resistance and central obesity both are considered significant factor for the development of Metabolic syndrome.15,16 other factors like genetics, physical inactivity, hormonal changes are not much significant and may vary on ethnicity. ${ }^{17,18}$ Intrahepetic effects of Insulin includes increased the rate of triglyceride synthesis. In insulin resistant patient free fatty acid present in high concentration with excess storage of triglyceride that excess triglyceride in secreted as VLDL. ${ }^{19}$ Metabolic syndrome is associated with this increased VLDL. Oxidative stress is another mechanism to explain metabolic syndrome. ${ }^{20}$ The onset of Metabolic syndrome is highly dependent on oxidative stress that's finally leads to type 2 diabetes mellitus and cardiovascular diseases. ${ }^{21}$ Disturbance in glucose metabolism, dyslipidaemia, hypertension and abdominal obesity are most common manifestations of Metabolic syndrome. Oxidative stress plays important role for its development. Accumulation of plasma free fatty acid increases the production of Reactive Oxygen Species by stimulating NOX and decreasing the antioxidant enzyme activity. ${ }^{22}$ There are many literatures available confirm the impairment of lipid and glucose metabolism in Vitiligo patients. In our study we did not find any significant difference in plasma glucose level when compared cases to control which is almost similar to study of Atas $\mathrm{H}$ et al Serum Triacylglycerol including LDL level were significantly increased when compared to control. ${ }^{23}$ Sharma et al, Atlas et al reported the same. 24, 25 Serum HDL is significantly decreased when compared the both groups. Deranging of lipid parameters possibly explained by lipid peroxidation theory linked with Vitiligo. ${ }^{26}$ There was no significant differences were observed in systolic, diastolic blood pressure and waist circumference in our study.

\section{CONCLUSIONS}

Metabolic syndrome is somehow related with Vitiligo. Literature in this regard is less. Further study is required to establish the relation between Vitiligo and Metabolic Syndrome. 


\section{REFERENCES}

[1] Prasad H, Ryan DA, Celzo MF, et al. Metabolic syndrome: definition and therapeutic implications. Postgrad Med 2012;124(1):21-30.

[2] PraveenKumar U, Ganguly S, Ray L, et al. Prevalence of metabolic syndrome in psoriasis patients and its relation to disease duration: a hospital based case - control study. J Clin Disgn Res 2016;10(2):WC01-WC05.

[3] Pietrzak A, Bartosinska J, Hercogova J, et al. Metabolic syndrome in vitiligo. Dermatol Ther 2012;25(Suppl 1):41-4.

[4] Touyz RM. Molecular and cellular mechanisms in vascular injury in hypertension: role of angiotensin II. Editorial review. Curr Opin Nephrol Hypertens 2005;14(2):125-31.

[5] Mueller CF, Laude K, McNally JS, et al. ATVB in focus: redox mechanisms in blood vessels. Arterioscler Thromb Vasc Biol 2005;25(2):274-8.

[6] Wu H, Yin JJ, Wamer WG, et al. Reactive oxygen speciesrelated activities of nano-iron metal and nano-iron oxides. J Food Drug Anal 2014;22(1):86-94.

[7] Alsufyani MA, Golant AK, Lebwohl M. Psoriasis and The metabolic syndrome. Dermatologic Therapy 2010;23(2):137-43.

[8] Agrawal D, Sahani MH, Gupta S. Vitiligo etiopathogenesis and therapy - a review. J MSUBB 2001;48:97-106.

[9] Thomas GN, Ho SY, Janus ED, et al. The US National Cholesterol Education Programme Adult Treatment Panel III (NCEP ATP III) prevalence of the metabolic syndrome in a Chinese population. Diabetes Res Clin Pract 2005;67(3):251-7.

[10] Ezzedine K, Lim HW, Suzuki T, et al. Revised classification/nomenclature of vitiligo and related issues: The Vitiligo Global Issues Consensus Conference. Pigment Cell Melanoma Res 2012;25(3):E1-13.

[11] Pinnell SR. Cutaneous photodamage, oxidative stress and topical antioxidant protection. J Am Acad Dermatol 2003;48(1):1-22.

[12] Jimbow K, Chen H, Park JS, et al. Increased sensitivity of melanocytes to oxidative stress and abnormal expression of tyrosinase-related protein in vitiligo. $\mathrm{Br} \mathrm{J}$ Dermatol 2001;144(1):55-65.

[13] Eskandani M, Golchai J, Pirooznia N, et al. Oxidative stress level and tyrosinase activity in vitiligo patients. Indian J Dermatol 2010;55(1):15-9.
[14] Maresca V, Roccella M, Roccella F, et al. Increased sensitivity to peroxidative agents as a possible pathogenic factor of melanocyte damage in vitiligo. J Invest Dermatol 1997;109(3):310-3.

[15] Hu G, Qiao Q, Tuomilehto J, et al. Plasma insulin and cardiovascular mortality in non-diabetic European men and women: a meta-analysis of data from eleven prospective studies. The DECODE Insulin Study Group. Diabetologia 2004;47(7):1245-56.

[16] Carr DB, Utzschneider KM, Hull RL, et al. Intra-abdominal fat is a major determinant of the National Cholesterol Education Program Adult Treatment Panel III criteria for the metabolic syndrome. Diabetes 2004;53(8):2087-94.

[17] Saad MF, Lillioja S, Nyomba BL, et al. Racial differences in the relation between blood pressure and insulin resistance. New England Journal of Medicine 1991;324(11):733-9.

[18] Anderson PJ, Critchley JA, Chan JC, et al. Factor analysis of the metabolic syndrome: obesity vs insulin resistance as the central abnormality. International Journal of Obesity Relat Metab Disord 2001;25(12):1782-8.

[19] Lewis GF, Steiner G. Acute effects of insulin in the control of VLDL production in humans. Implications for the insulin-resistant state. Diabetes Care 1996;19(4):390-3.

[20] Zhou SS, Li D, Zhou YM, et al. The skin function: a factor of anti-metabolic syndrome. Diabetol Metab Syndr 2012;4(1):15.

[21] Spahis S, Borys JM, Levy E. Metabolic syndrome as a multifaceted risk factor for oxidative stress. Antioxidants \& Redox Signaling 2017;26(9):445-61.

[22] Matsuda M, Shimomura I. Increased oxidative stress in obesity: implications for metabolic syndrome, diabetes, hypertension, dyslipidaemia, atherosclerosis and cancer. Obesity Research \& Clinical Practice 2013;7(5):e330-41.

[23] Atas H, Gonul M. The increased risk of metabolic syndrome in patients with vitiligo. Balkan Med J 2017;34(3):219-25.

[24] Sharma YK, Bansal P, Menon S, et al. Metabolic syndrome in vitiligo patients among a semi urban Maharashtrian population: a case control study. Diab Met Syndr 2017;(11 Suppl 1):S77-S80.

[25] Karadag AS, Tutal E, Ertugrul DT. Insulin resistance is increased patients with vitiligo. Acta Derma Venereol 2011;91(5):541-4.

[26] Bhor U, Pande S. Scoring systems in dermatology. Indian J Dermatol Venereol Leprol 2006;72(4):315-21. 